\title{
Densities, Refractive Indices and Excess Properties of Binary Mixtures of Acetonitrile with Benzene, Toluene, $m$-Xylene and Mesitylene at Temperatures from (298.15 to 313.15) $\mathrm{K}$
}

\author{
Abeer K. Shams \\ Department of Chemistry, College of Science, Al-Nahrain University.
}

\begin{abstract}
The densities $(\rho)$ and refractive indices $\left(n_{\mathrm{D}}\right)$ of binary mixtures of acetonitrile with benzene, toluene, $m$-xylene and mesitylene including those of pure liquids, over the entire composition range expressed by mole fraction $x_{1}$ of acetonitrile were measured at temperatures $(298.15,303.15$, 308.15, and 313.15) K under atmospheric pressure. From the experimental data, the excess molar volumes $\left(V_{m}^{E}\right)$ and the deviations in refractive indices $(\Delta n)$ were calculated. These results are fit to a Redlich-Kister type polynomial equation. The results indicate the presence of weak interactions between acetonitrile and aromatic hydrocarbon molecules. The deviations in $V_{m}^{E}$ values follow the order toluene $<$ benzene $<m$-xylene $<$ mesitylene. The results are discussed in terms of the intermolecular interactions.

\section{Introduction}

Studies of physicochemical properties of binary mixtures are of considerable importance in the fundamental understanding of the nature of the interactions between unlike molecules. In recent years there has been

mixtures for acetonitrile + aromatic hydrocarbons. Afaf H. Absood [6] report densities of these binary systems at $303.15 \mathrm{~K}$. Nevertheless, to our knowledge, no literature data on densities and refractive indices are available for this system.
\end{abstract} considerable interest in theoretical and experimental investigations of the excess thermodynamic properties of binary mixtures [1-3]. Refractive index and density measurements are expected to shed some light on both solvent - solvent and solute - solvent interactions [4]. The excess properties of binary liquid mixtures are important for understanding and interpreting the interactions between molecules of a mixture [5].

In this work, The densities and refractive indices of binary mixtures of acetonitrile with benzene, toluene, $m$-xylene and mesitylene at temperatures $(298.15,303.15,308.15$, and 313.15) K were studied. The Redlich-Kister equation was used to correct and fit the experimental excess molar volumes and the refractive index deviations. The variation of these parameters with the composition and temperature of the mixtures has been discussed in terms of the molecular interactions in these mixtures. The effect of the number and position of the methyl groups in these aromatic hydrocarbons on molecular interactions in these mixtures has also been discussed.

A survey of the literature shows that very few measurements have been made on the densities and refractive indices of binary

\section{Experimental \\ Chemicals:}

Acetonitrile, mesitylene (Fluka AG, mole fraction purity > 0.998), benzene, toluene and $m$-xylene (Merck, mole fraction purity > 0.995) were used without further purification. All liquids were stored over freshly activated molecular sieves of type 4A (union Carbide). Doubly distilled water was used as the standard liquid with conductivity less than $0.05 \mu \mathrm{s} \mathrm{cm}^{-1}$.

\section{Apparatus and Procedure:}

The mixtures were prepared by mass and were kept in special airtight stopper glass bottles to avoid evaporation. The weightings were done using an electronic balance (model GR-202R, AND, Japan) with a precision of \pm $0.01 \mathrm{mg}$. The uncertainty in the mole fraction was estimated to be less than \pm 0.0001 .

The density measurements of the pure solvents and the mixtures were performed by means of an Anton Paar, model DMA 48, Graz, Austria with a precision of $\pm 0.00005 \mathrm{~g} \mathrm{~cm}^{-3}$, between 298.15 and 313.15 K. The DMA cell was calibrated with dry air and doubly distilled water at atmospheric pressure. The sample size was $0.70 \mathrm{~cm}^{3}$ 
and the sample thermostat was controlled to $\pm 0.01 \mathrm{~K}$. Triplicate measurements of the density were performed for all the mixtures and pure components. The accuracy in the determination of the density is believed to be less than $\pm 1 \times 10^{-2} \mathrm{~kg} \cdot \mathrm{m}^{-3}$.

The refractive indices of pure liquids and their binary mixture were measured using a thermostatted Abbe refractometer (Tefsa). We calibrated the refractometer by measuring the refractive indices of doubly distilled water and toluene at various temperatures. The values of refractive indices were obtained using sodium $\mathrm{D}$ light. The temperature of the test liquids between the prisms of the refractometer during the measurements was maintained to an uncertainty of $\pm 0.01 \mathrm{~K}$ by circulating water through the jacket around the prisms from an electronically controlled thermostatic water bath, and the temperature was measured with a digital thermometer connected to the prism jacket. The uncertainty in refractive index measurements was within \pm 0.0001 . The temperature of the test liquids during the measurements was maintained to an uncertainty of $\pm 0.01 \mathrm{~K}$ in an electronically controlled thermostatic water bath (a HAKKED1-G Germany). The reliability of experimental measurements of $\rho$ and $n_{\mathrm{D}}$ was ascertained by a comparison of the experimental data of pure liquids with the corresponding literature values at $298.15 \mathrm{~K}$ (Table (1)), and the agreement between the values was found to be good.

Table (1)

Comparison of experimental values of density $\rho$ and refractive index $n_{D}$ of pure liquids with the corresponding literature values at $298.15 \mathrm{~K}$.

\begin{tabular}{|c|c|c|c|c|c|c|}
\hline \multirow{2}{*}{ Substance } & \multicolumn{3}{|c|}{$\rho / g . \mathrm{cm}^{-3}$} & \multicolumn{3}{|c|}{$n_{D}$} \\
\hline & Exptl. & Lit. & ref & Exptl. & Lit. & ref \\
\hline Acetonitrile & 0.77660 & $\begin{array}{l}0.77640 \\
0.77658 \\
\end{array}$ & $\begin{array}{l}7 \\
8 \\
\end{array}$ & 1.3420 & $\begin{array}{l}1.3429 \\
1.3422 \\
\end{array}$ & $\begin{array}{l}9 \\
8 \\
\end{array}$ \\
\hline Benzene & 0.87381 & $\begin{array}{l}0.87360 \\
0.87362\end{array}$ & $\begin{array}{l}10 \\
11 \\
\end{array}$ & 1.4980 & $\begin{array}{c}1.49792 \\
1.4979\end{array}$ & $\begin{array}{l}10 \\
12\end{array}$ \\
\hline Toluene & 0.8621 & $\begin{array}{l}0.86219 \\
0.86231\end{array}$ & $\begin{array}{l}10 \\
13 \\
\end{array}$ & 1.4942 & $\begin{array}{c}1.49413 \\
1.4941\end{array}$ & $\begin{array}{l}10 \\
12\end{array}$ \\
\hline m-Xylene & 0.8599 & $\begin{array}{l}0.8599 \\
0.8597\end{array}$ & $\begin{array}{l}10 \\
14\end{array}$ & 1.4946 & $\begin{array}{l}1.4948 \\
1.4945\end{array}$ & $\begin{array}{l}15 \\
16\end{array}$ \\
\hline Mesitylene & 0.8614 & 0.8614 & 17 & 1.4967 & 1.4969 & 17 \\
\hline
\end{tabular}

\section{Results and Discussion}

The experimental densities $(\rho)$, refractive indices $\left(n_{\mathrm{D}}\right)$, the excess molar volumes $\left(V_{m}^{E}\right)$ and deviations in the refraction $(\Delta n)$ of binary mixtures of acetonitrile with benzene, toluene, $m$-xylene and mesitylene at various mole fractions and at the four temperatures are listed in Tables (2-5). The excess molar volumes $\left(V_{m}^{E}\right)$ and deviations in the refraction $(\Delta n)[18]$ of the mixtures were calculated using the following relations:

$V_{m}^{E}=\chi M_{1}\left(\frac{1}{\rho}-\frac{1}{\rho_{1}}\right)+(1-\chi) M_{2}\left(\frac{1}{\rho}-\frac{1}{\rho_{2}}\right) \ldots$

$\Delta n=n-\left[\phi n_{1}+(1-\phi) n_{2}\right]$

Where $\mathrm{M}$ is the molar mass, $\varnothing$ is the volume fraction (calculated using the molar volumes of the pure components obtained from the density data), and subscripts 1 and 2 stand for pure components acetonitrile and aromatic hydrocarbons, respectively.

The $V_{m}^{E}$ and $\Delta n$ values were fitted to a Redlich-Kister-type[19] polynomial equation:

$Y=\chi_{i} \chi_{j} \sum_{K=0}^{P} A_{K}\left(\chi_{i}-\chi_{j}\right)^{K}$

Where $\mathrm{Y}$ is $V_{m}^{E}$ or $\Delta n$ and $\mathrm{p}$ is the degree of polynomial expansion. The standard deviations were calculated by means of the equation [20]:

$\sigma=\left[\frac{\sum_{i=1}^{n}\left(Y_{i}{ }^{\text {exp }}-Y_{i}^{\text {calc }}\right)^{2}}{n-m}\right]^{0.5}$

Where $n$ is the number of experimental data and $m$ is the number of parameters. The values of coefficients, $A_{k}$ were evaluated by using the method of least-squares with all points 
weighted equally. The coefficients $\mathrm{A}_{0}, \mathrm{~A}_{1}, \mathrm{~A}_{2}$ and $\mathrm{A}_{3}$ of $V_{m}^{E}$ and $\Delta n$ for the mixtures at all investigated temperatures and the corresponding standard deviations are listed in

\begin{tabular}{|c|c|c|c|c|}
\hline$x_{1}$ & $\rho /$ g. $\mathrm{cm}^{-3}$ & $n_{D}$ & $V_{m}^{E} / \mathrm{cm}^{3} \mathrm{~mol}^{-1}$ & $\Delta \mathrm{n} / \mathrm{cm}^{3} \mathrm{~mol}^{-1}$ \\
\hline \multicolumn{5}{|c|}{$T=298.15 \mathrm{~K}$} \\
\hline 0.0000 & 0.87381 & 1.4980 & 0.000 & 0.000 \\
\hline 0.0939 & 0.87004 & 1.4935 & -0.183 & -0.485 \\
\hline 0.1933 & 0.86464 & 1.4811 & $\begin{array}{l}-0.277 \\
\end{array}$ & -0.972 \\
\hline 0.2916 & 0.85804 & 1.4698 & -0.300 & -1.368 \\
\hline 0.3873 & 0.85053 & 1.4576 & -0.281 & -1.654 \\
\hline 0.4979 & 0.84062 & 1.4412 & -0.233 & -1.871 \\
\hline 0.5866 & 0.83175 & 1.4262 & -0.188 & $\begin{array}{l}-1.939 \\
\end{array}$ \\
\hline 0.7024 & 0.81884 & 1.4039 & -0.130 & -1.852 \\
\hline 0.8064 & 0.80581 & 1.3837 & -0.083 & -1.482 \\
\hline 0.8952 & 0.79331 & 1.3653 & -0.046 & -0.932 \\
\hline 1.0000 & 0.77660 & 1.3420 & 0.000 & 0.000 \\
\hline \multicolumn{5}{|c|}{$\mathrm{T}=303.15 \mathrm{~K}$} \\
\hline 0.0000 & 0.86826 & 1.4949 & 0.000 & 0.000 \\
\hline 0.0939 & 0.86475 & 1.4885 & -0.195 & $\begin{array}{c}-0.461 \\
\end{array}$ \\
\hline 0.1933 & 0.85962 & 1.4777 & -0.299 & -0.997 \\
\hline 0.2916 & 0.85332 & 1.4668 & -0.332 & -1.393 \\
\hline 0.3873 & 0.84614 & 1.4550 & -0.324 & -1.677 \\
\hline 0.4979 & 0.83663 & 1.4395 & -0.287 & -1.878 \\
\hline 0.5866 & 0.82806 & 1.4245 & -0.246 & -1.959 \\
\hline 0.7024 & 0.81545 & 1.4025 & -0.184 & -1.877 \\
\hline 0.8064 & 0.80257 & 1.3829 & -0.123 & -1.496 \\
\hline 0.8952 & 0.79012 & 1.3649 & -0.067 & -0.944 \\
\hline 1.0000 & 0.77350 & 1.3412 & 0.000 & 0.000 \\
\hline \multicolumn{5}{|c|}{$\mathrm{T}=308.15 \mathrm{~K}$} \\
\hline 0.0000 & 0.86290 & 1.4918 & 0.000 & 0.000 \\
\hline 0.0939 & 0.85935 & 1.4851 & -0.214 & -0.491 \\
\hline 0.1933 & 0.85432 & $\begin{array}{l}1.4744 \\
\end{array}$ & -0.350 & -1.035 \\
\hline 0.2916 & 0.84805 & ב1.4634 & -0.408 & -1.439 \\
\hline 0.3873 & 0.84075 & 1.4515 & $\begin{array}{c}-0.409 \\
\end{array}$ & -1.733 \\
\hline 0.4979 & 0.83089 & 1.4357 & -0.365 & -1.946 \\
\hline 0.5866 & 0.82192 & 1.4204 & -0.310 & -2.038 \\
\hline $\begin{array}{l}0.7024 \\
\end{array}$ & 0.80873 & 1.3980 & $\begin{array}{l}-0.229 \\
\end{array}$ & -1.972 \\
\hline 0.8064 & 0.79536 & 1.3781 & -0.156 & -1.606 \\
\hline 0.8952 & 0.78246 & 1.3596 & $\begin{array}{l}-0.091 \\
\end{array}$ & $\begin{array}{l}-0.491 \\
\end{array}$ \\
\hline 1.0000 & 0.76511 & 1.3404 & 0.000 & 0.000 \\
\hline \multicolumn{5}{|c|}{$\mathrm{T}=313.15 \mathrm{~K}$} \\
\hline $\begin{array}{l}0.0000 \\
\end{array}$ & 0.85601 & 1.4887 & 0.000 & 0.000 \\
\hline 0.0939 & 0.85276 & 1.4843 & -0.232 & -0.552 \\
\hline 0.1933 & 0.84798 & 1.4737 & -0.375 & -1.087 \\
\hline 0.2916 & 0.84195 & 1.4628 & -0.436 & -1.482 \\
\hline 0.3873 & 0.83490 & 1.4510 & -0.439 & -1.765 \\
\hline 0.4979 & 0.82536 & 1.4352 & -0.397 & -1.965 \\
\hline 0.5866 & 0.81662 & 1.4199 & "-0.341 & "-2.044 \\
\hline 0.7024 & 0.80376 & 1.3973 & -0.256 & -1.961 \\
\hline 0.8064 & 0.79062 & 1.3770 & -0.173 & $\begin{array}{l}-1.579 \\
\end{array}$ \\
\hline 0.8952 & 0.77794 & 1.3581 & -0.099 & -1.025 \\
\hline 1.0000 & 0.76091 & 1.3361 & 0.000 & 0.000 \\
\hline
\end{tabular}

Tables (6). The variations of $V_{m}^{E}$ and $\Delta n$ with mole fraction $x_{1}$ of acetonitrile at $298.15 \mathrm{~K}$ are presented graphically in Figs. (1 and 2).

\section{Table (2)
Densities, refractive index, excess molar volumes, and deviations in molar refraction for the \\ Table (2)
Densities, refractive index, excess molar volumes, and deviations in molar refraction for the acetonitrile $(1)+$ benzene (2) binary mixtures at the temperatures $(298.15$ to 313.15)K.}


Table (3)

Densities, refractive index, excess molar volumes, and deviations in molar refraction for the acetonitrile $(1)+$ toluene (2) binary mixtures at the temperatures $(298.15$ to 313.15) K.

\begin{tabular}{|c|c|c|c|c|}
\hline$x_{1}$ & $\rho /$ g. $\mathrm{cm}^{-3}$ & $n_{D}$ & $V_{m}^{E} / \mathrm{cm}^{3} \mathrm{~mol}^{-1}$ & $\Delta n / \mathrm{cm}^{3} \mathrm{~mol}^{-1}$ \\
\hline \multicolumn{5}{|c|}{$T=298.15 \mathrm{~K}$} \\
\hline 0.0000 & 0.86210 & 1.4942 & 0.000 & 0.000 \\
\hline 0.0726 & 0.86066 & 1.4921 & -0.209 & -0.570 \\
\hline 0.1866 & 0.85679 & 1.4835 & -0.385 & -1.530 \\
\hline 0.3040 & 0.85111 & 1.4692 & -0.447 & -2.507 \\
\hline 0.4021 & 0.84437 & 1.4566 & -0.443 & -3.078 \\
\hline 0.4736 & 0.84001 & 1.4477 & -0.410 & -3.335 \\
\hline 0.6042 & 0.82905 & 1.4273 & -0.335 & -3.543 \\
\hline 0.6878 & 0.82079 & 1.4126 & -0.278 & -3.405 \\
\hline 0.7809 & 0.81006 & 1.3957 & -0.201 & -2.890 \\
\hline 0.0954 & 0.79454 & 1.3697 & $\begin{array}{l}-0.101 \\
\end{array}$ & -1.793 \\
\hline 1.0000 & 0.77660 & 1.3420 & 0.000 & 0.000 \\
\hline \multicolumn{5}{|c|}{$\mathrm{T}=303.15 \mathrm{~K}$} \\
\hline 0.0000 & 0.85730 & 1.4913 & 0.000 & 0.000 \\
\hline 0.0726 & 0.85591 & 1.4873 & -0.205 & -0.668 \\
\hline 0.1866 & 0.85222 & 1.4778 & -0.390 & -1.682 \\
\hline 0.3040 & 0.84676 & 1.4665 & -0.462 & -2.515 \\
\hline 0.4021 & 0.84017 & 1.4538 & -0.458 & -3.102 \\
\hline 0.4736 & 0.83589 & 1.4458 & -0.425 & -3.329 \\
\hline 0.6042 & 0.82517 & 1.4266 & -0.350 & -3.505 \\
\hline 0.6878 & 0.81706 & 1.4128 & -0.292 & -3.342 \\
\hline 0.7809 & 0.80654 & 1.3930 & -0.215 & -2.940 \\
\hline 0.0954 & 0.79126 & 1.3680 & -0.111 & -1.816 \\
\hline 1.0000 & 0.77350 & 1.3412 & 0.000 & 0.000 \\
\hline \multicolumn{5}{|c|}{$\mathrm{T}=308.15 \mathrm{~K}$} \\
\hline 0.0000 & 0.852911 & 1.4883 & 0.000 & 0.000 \\
\hline 0.0726 & 0.85133 & 1.4840 & -0.210 & -0.679 \\
\hline 0.1866 & 0.84751 & 1.4778 & -0.415 & -1.566 \\
\hline 0.3040 & 0.84173 & 1.4636 & -0.487 & -2.501 \\
\hline 0.4021 & 0.83479 & 1.4517 & -0.483 & -3.049 \\
\hline 0.4736 & 0.83037 & 1.4424 & -0.455 & -3.333 \\
\hline 0.6042 & 0.81912 & 1.4237 & -0.375 & -3.492 \\
\hline 0.6878 & 0.81072 & 1.4091 & -0.322 & -3.366 \\
\hline 0.7809 & 0.79972 & 1.3921 & -0.240 & -2.876 \\
\hline 0.0954 & 0.78366 & 1.3404 & -0.121 & -1.798 \\
\hline 1.0000 & 0.76511 & 1.3404 & 0.000 & 0.000 \\
\hline \multicolumn{5}{|c|}{$\mathrm{T}=313.15 \mathrm{~K}$} \\
\hline 0.0000 & 0.84821 & 1.4858 & 0.000 & 0.000 \\
\hline 0.0726 & 0.84668 & 1.4836 & -0.215 & -0.568 \\
\hline 0.1866 & 0.84296 & 1.4733 & -0.430 & -1.627 \\
\hline 0.3040 & 0.83718 & 1.4650 & -0.499 & -2.335 \\
\hline 0.4021 & 0.83033 & 1.4525 & -0.498 & -2.888 \\
\hline 0.4736 & 0.82596 & 1.4428 & -0.472 & -3.205 \\
\hline 0.6042 & 0.81479 & 1.4243 & -0.392 & -3.355 \\
\hline 0.6878 & 0.80642 & 1.4109 & -0.337 & -3.188 \\
\hline 0.7809 & 0.79550 & 1.3905 & -0.255 & -2.820 \\
\hline 0.0954 & 0.77953 & 1.3652 & -0.135 & -1.722 \\
\hline 1.0000 & 0.76091 & 1.3361 & 0.000 & 0.000 \\
\hline
\end{tabular}


Table (4)

Densities, refractive index, excess molar volumes, and deviations in molar refraction for the acetonitrile $(1)+m$-xylene (2) binary mixtures at the temperatures $(298.15$ to 313.15$) \mathrm{K}$.

\begin{tabular}{|c|c|c|c|c|}
\hline$x_{1}$ & $\rho /$ g. $\mathbf{c m}^{-3}$ & $n_{\mathrm{D}}$ & $V_{m}^{E} / \mathrm{cm}^{3} \mathrm{~mol}^{-1}$ & $\Delta n / \mathrm{cm}^{3} \mathrm{~mol}^{-1}$ \\
\hline \multicolumn{5}{|c|}{$\mathrm{T}=298.15$} \\
\hline 0.0000 & 0.85990 & 1.4946 & 0.000 & 0.000 \\
\hline 0.1307 & 0.85582 & 1.4889 & -0.128 & -1.545 \\
\hline 0.1904 & 0.85360 & 1.4836 & -0.171 & -2.302 \\
\hline 0.2770 & 0.84974 & 1.4766 & -0.193 & -3.190 \\
\hline 0.3779 & 0.84438 & 1.4677 & -0.192 & -4.011 \\
\hline 0.4800 & 0.83791 & 1.4560 & -0.172 & -4.641 \\
\hline 0.5938 & 0.82920 & 1.4400 & -0.135 & -4.961 \\
\hline 0.7057 & 0.81883 & 1.4201 & -0.102 & -4.785 \\
\hline 0.8033 & 0.80774 & 1.4004 & "-0.069 & -3.998 \\
\hline 0.8926 & 0.79541 & $\begin{array}{l}1.3744 \\
\end{array}$ & -0.041 & -2.781 \\
\hline 1.0000 & 0.77660 & 1.3420 & 0.000 & 0.000 \\
\hline \multicolumn{5}{|c|}{$\mathrm{T}=303.15 \mathrm{~K}$} \\
\hline 0.0000 & 0.85530 & 1.4932 & 0.000 & 0.000 \\
\hline 0.1307 & 0.85141 & 1.4876 & -0.142 & -1.556 \\
\hline 0.1904 & 0.84920 & 1.4822 & -0.179 & -2.316 \\
\hline 0.2770 & 0.84541 & 1.4754 & -0.201 & -3.204 \\
\hline 0.3779 & 0.84015 & 1.4661 & -0.199 & -4.052 \\
\hline 0.4800 & 0.83379 & 1.4550 & -0.177 & -4.653 \\
\hline 0.5938 & 0.82525 & 1.4386 & -0.141 & -5.001 \\
\hline 0.7057 & 0.81508 & $\begin{array}{l}1.4184 \\
\end{array}$ & $\begin{array}{l}-0.108 \\
\end{array}$ & -4.831 \\
\hline 0.8033 & 0.80417 & 1.3996 & -0.074 & -4.012 \\
\hline 0.8926 & 0.79201 & 1.3738 & -0.042 & -2.785 \\
\hline 1.0000 & 0.77350 & 1.3412 & 0.000 & 0.000 \\
\hline \multicolumn{5}{|c|}{$\mathrm{T}=308.15 \mathrm{~K}$} \\
\hline 0.0000 & 0.85240 & 1.4916 & 0.000 & 0.000 \\
\hline 0.1307 & 0.84815 & 1.4848 & -0.145 & -1.610 \\
\hline 0.1904 & 0.84577 & 1.4801 & -0.184 & -2.323 \\
\hline 0.2770 & 0.84169 & 1.4722 & -0.207 & -3.259 \\
\hline 0.3779 & 0.83604 & 1.4621 & -0.205 & -4.131 \\
\hline 0.4800 & 0.82926 & 1.4516 & -0.185 & -4.688 \\
\hline 0.5938 & 0.82013 & 1.4331 & -0.147 & -5.110 \\
\hline 0.7057 & 0.80929 & 1.4124 & -0.115 & -4.951 \\
\hline 0.8033 & 0.79767 & 1.3919 & -0.079 & -4.190 \\
\hline 0.8926 & 0.78473 & 1.3715 & -0.145 & -2.786 \\
\hline 1.0000 & 0.76511 & 1.3404 & 0.000 & 0.000 \\
\hline \multicolumn{5}{|c|}{$\mathrm{T}=313.15 \mathrm{~K}$} \\
\hline 0.0000 & 0.84730 & 1.4886 & 0.000 & 0.000 \\
\hline 0.1307 & 0.84323 & 1.4817 & -0.151 & -1.612 \\
\hline 0.1904 & 0.84087 & 1.4768 & -0.19 & -2.346 \\
\hline 0.2770 & 0.83684 & 1.4700 & -0.213 & -3.223 \\
\hline 0.3779 & 0.83125 & 1.4601 & -0.211 & -4.088 \\
\hline 0.4800 & 0.82453 & 1.4509 & -0.191 & -4.585 \\
\hline 0.5938 & 0.81554 & 1.4320 & -0.158 & -5.034 \\
\hline 0.7057 & 0.80476 & 1.4114 & -0.121 & -4.872 \\
\hline 0.8033 & 0.79325 & 1.3889 & -0.085 & -4.18 \\
\hline 0.8926 & 0.78037 & 1.3680 & -0.045 & -2.782 \\
\hline 1.0000 & 0.76091 & 1.3361 & 0.000 & 0.000 \\
\hline
\end{tabular}


Table (5)

Densities, refractive index, excess molar volumes, and deviations in molar refraction for the acetonitrile(1) + mesitylene (2) binary mixtures at the temperatures (298.15 to 313.15) $\mathrm{K}$.

\begin{tabular}{|c|c|c|c|c|}
\hline$x_{1}$ & $\rho / g \cdot \mathrm{cm}^{-3}$ & $n_{D}$ & $V_{m}^{E} / \mathrm{cm}^{3} \mathrm{~mol}^{-1}$ & $\Delta \mathrm{n} / \mathrm{cm}^{3} \mathrm{~mol}^{-1}$ \\
\hline \multicolumn{5}{|c|}{$\mathrm{T}=298.15$} \\
\hline 0.0000 & 0.86140 & 1.4967 & 0.000 & 0.000 \\
\hline 0.0972 & 0.85771 & 1.4881 & 0.055 & -1.850 \\
\hline 0.1924 & 0.85371 & 1.4849 & 0.095 & -3.120 \\
\hline 0.2972 & 0.84878 & 1.4778 & 0.121 & -4.472 \\
\hline 0.4062 & 0.84288 & 1.4661 & 0.131 & -5.766 \\
\hline 0.5005 & 0.83695 & 1.4552 & 0.128 & -6.512 \\
\hline 0.6179 & 0.82807 & 1.4387 & 0.115 & -6.905 \\
\hline 0.7146 & 0.81914 & 1.4207 & 0.092 & -6.676 \\
\hline 0.8192 & 0.80706 & 1.3990 & 0.064 & -5.468 \\
\hline 0.8912 & 0.79673 & 1.3798 & 0.042 & -3.938 \\
\hline 1.0000 & 0.77660 & 1.3420 & 0.000 & 0.000 \\
\hline \multicolumn{5}{|c|}{$\mathrm{T}=303.15 \mathrm{~K}$} \\
\hline 0.0000 & 0.85710 & 1.4946 & 0.000 & 0.000 \\
\hline 0.0972 & 0.85345 & 1.4922 & 0.057 & -1.447 \\
\hline 0.1924 & 0.84949 & 1.4868 & 0.100 & -2.884 \\
\hline 0.2972 & 0.84462 & 1.4768 & 0.127 & -4.439 \\
\hline 0.4062 & 0.83880 & 1.4650 & 0.138 & -5.739 \\
\hline 0.5005 & 0.83295 & 1.4542 & 0.135 & -6.490 \\
\hline 0.6179 & 0.82419 & 1.4382 & 0.122 & -6.872 \\
\hline 0.7146 & 0.81535 & 1.4202 & 0.102 & -6.652 \\
\hline 0.8192 & 0.80347 & 1.3987 & 0.070 & -5.447 \\
\hline 0.8912 & 0.79326 & 1.3783 & 0.049 & -3.961 \\
\hline 1.0000 & 0.77350 & 1.3412 & 0.000 & 0.000 \\
\hline \multicolumn{5}{|c|}{$\mathrm{T}=308.15 \mathrm{~K}$} \\
\hline 0.0000 & 0.85280 & 1.4922 & 0.000 & 0.000 \\
\hline 0.0972 & 0.84893 & 1.4878 & 0.065 & -1.568 \\
\hline 0.1924 & 0.84474 & 1.4827 & 0.112 & -2.969 \\
\hline 0.2972 & 0.83962 & 1.4740 & 0.139 & -4.434 \\
\hline 0.4062 & 0.83348 & 1.4636 & 0.151 & -5.650 \\
\hline 0.5005 & 0.82731 & 1.4528 & 0.150 & -6.400 \\
\hline 0.6179 & 0.81810 & 1.4322 & 0.136 & -6.813 \\
\hline 0.7146 & 0.80881 & 1.4173 & 0.116 & -6.637 \\
\hline 0.8192 & 0.79631 & 1.3947 & 0.085 & -5.481 \\
\hline 0.8912 & 0.78571 & 1.3754 & 0.055 & -3.974 \\
\hline 1.0000 & 0.76511 & 1.3404 & 0.000 & 0.000 \\
\hline \multicolumn{5}{|c|}{$\mathrm{T}=313.15 \mathrm{~K}$} \\
\hline 0.0000 & 0.84880 & 1.4893 & 0.000 & 0.000 \\
\hline 0.0972 & 0.84489 & 1.4858 & 0.070 & -1.504 \\
\hline 0.1924 & 0.84068 & 1.4798 & 0.119 & -2.961 \\
\hline 0.2972 & 0.83553 & 1.4701 & 0.148 & -4.482 \\
\hline 0.4062 & 0.82938 & 1.4593 & 0.159 & -5.712 \\
\hline 0.5005 & 0.82318 & 1.4501 & 0.158 & -6.372 \\
\hline 0.6179 & 0.81392 & 1.4325 & 0.147 & -6.882 \\
\hline 0.7146 & 0.80459 & 1.4146 & 0.127 & -6.600 \\
\hline 0.8192 & 0.79205 & 1.3926 & 0.096 & -5.418 \\
\hline 0.8912 & 0.78141 & 1.3715 & 0.067 & -3.970 \\
\hline 1.0000 & 0.76091 & 1.3361 & 0.000 & 0.000 \\
\hline
\end{tabular}


Table (6)

Estimated Parameters of Excess and Deviation Funtions for Mixtures.

\begin{tabular}{|c|c|c|c|c|c|c|}
\hline Function & $T / K$ & $A_{0}$ & $A_{1}$ & $A_{2}$ & $A_{3}$ & $\sigma$ \\
\hline \multicolumn{7}{|c|}{ acetonitrile(1) + benzene (2) } \\
\hline$V_{m}^{E} / \mathrm{cm}^{3} \mathrm{~mol}^{-1}$ & $\begin{array}{l}298.15 \\
303.15 \\
308.15 \\
313.15 \\
\end{array}$ & $\begin{array}{l}-0.9302 \\
-1.1481 \\
-1.4586 \\
-1.5835 \\
\end{array}$ & $\begin{array}{l}-0.9967 \\
-0.8496 \\
-1.1064 \\
-1.0876 \\
\end{array}$ & $\begin{array}{l}-0.6026 \\
-0.5427 \\
-0.4382 \\
-0.4674 \\
\end{array}$ & $\begin{array}{c}-0.0456 \\
-0.1910 \\
0.2443 \\
0.0861 \\
\end{array}$ & $\begin{array}{l}0.002 \\
0.002 \\
0.001 \\
0.002 \\
\end{array}$ \\
\hline$\Delta n / \mathrm{cm}^{3} \mathrm{~mol}^{-1}$ & $\begin{array}{l}298.15 \\
303.15 \\
308.15 \\
313.15 \\
\end{array}$ & $\begin{array}{l}-7.5488 \\
-7.6355 \\
-7.8794 \\
-7.9521 \\
\end{array}$ & $\begin{array}{l}2.7045 \\
2.5819 \\
2.7469 \\
2.5875 \\
\end{array}$ & $\begin{array}{l}-0.7335 \\
-0.7141 \\
-1.4798 \\
-1.4950 \\
\end{array}$ & $\begin{array}{c}-0.0962 \\
0.3422 \\
1.0436 \\
0.1938 \\
\end{array}$ & $\begin{array}{l}0.014 \\
0.025 \\
0.018 \\
0.016 \\
\end{array}$ \\
\hline \multicolumn{7}{|c|}{ acetonitrile $(1)+$ toluene $(2)$} \\
\hline$V_{m}^{E} / \mathrm{cm}^{3} \mathrm{~mol}^{-1}$ & $\begin{array}{l}298.15 \\
303.15 \\
308.15 \\
313.15 \\
\end{array}$ & $\begin{array}{l}-1.5980 \\
-1.6630 \\
-1.7783 \\
-1.8399 \\
\end{array}$ & $\begin{array}{l}-1.0384 \\
-1.0591 \\
-1.0046 \\
-1.0434\end{array}$ & $\begin{array}{l}-0.6264 \\
-0.6113 \\
-0.6562 \\
-0.7352\end{array}$ & $\begin{array}{l}-0.2184 \\
-0.0478 \\
-0.0799 \\
-0.0359\end{array}$ & $\begin{array}{l}0.002 \\
0.002 \\
0.004 \\
0.004\end{array}$ \\
\hline$\Delta n / \mathrm{cm}^{3} \mathrm{~mol}^{-1}$ & $\begin{array}{l}298.15 \\
303.15 \\
308.15 \\
313.15 \\
\end{array}$ & $\begin{array}{l}-13.7508 \\
-13.6120 \\
-13.5790 \\
-12.9547 \\
\end{array}$ & $\begin{array}{l}4.9303 \\
4.5137 \\
4.8811 \\
4.6774 \\
\end{array}$ & $\begin{array}{l}-0.2428 \\
-1.8124 \\
-1.1192 \\
-1.4944 \\
\end{array}$ & $\begin{array}{l}2.1818 \\
1.8764 \\
1.3124 \\
1.4316 \\
\end{array}$ & $\begin{array}{l}0.019 \\
0.018 \\
0.028 \\
0.043 \\
\end{array}$ \\
\hline \multicolumn{7}{|c|}{ acetonitrile $(1)+\mathrm{m}$-xylene $(2)$} \\
\hline$V_{m}^{E} / \mathrm{cm}^{3} \mathrm{~mol}^{-1}$ & $\begin{array}{l}298.15 \\
303.15 \\
308.15 \\
313.15 \\
\end{array}$ & $\begin{array}{l}-0.6663 \\
-0.6878 \\
-0.7168 \\
-0.7476 \\
\end{array}$ & $\begin{array}{l}-0.5861 \\
-0.5697 \\
-0.5649 \\
-0.5335 \\
\end{array}$ & $\begin{array}{l}-0.2433 \\
-0.3142 \\
-0.3151 \\
-0.3258 \\
\end{array}$ & $\begin{array}{c}0.1717 \\
0.0368 \\
0.0235 \\
-0.0770 \\
\end{array}$ & $\begin{array}{l}0.002 \\
0.001 \\
0.002 \\
0.001 \\
\end{array}$ \\
\hline$\Delta n / \mathrm{cm}^{3} \mathrm{~mol}^{-1}$ & $\begin{array}{l}298.15 \\
303.15 \\
308.15 \\
313.15 \\
\end{array}$ & $\begin{array}{l}-18.9098 \\
-19.0459 \\
-19.3777 \\
-19.0210\end{array}$ & $\begin{array}{l}7.7714 \\
7.9267 \\
8.6970 \\
8.4513\end{array}$ & $\begin{array}{l}-3.5441 \\
-3.4717 \\
-3.8391 \\
-4.6381\end{array}$ & $\begin{array}{l}3.1023 \\
2.7670 \\
1.6398 \\
1.8571\end{array}$ & $\begin{array}{l}0.031 \\
0.034 \\
0.033 \\
0.030\end{array}$ \\
\hline \multicolumn{7}{|c|}{ acetonitrile $(1)+$ mesitylene $(2)$} \\
\hline$V_{m}^{E} / \mathrm{cm}^{3} \mathrm{~mol}^{-1}$ & $\begin{array}{l}298.15 \\
303.15 \\
308.15 \\
313.15 \\
\end{array}$ & $\begin{array}{l}0.5149 \\
0.5440 \\
0.6003 \\
0.6344\end{array}$ & $\begin{array}{l}0.1553 \\
0.1474 \\
0.1255 \\
0.1170\end{array}$ & $\begin{array}{l}0.2100 \\
0.1493 \\
0.1032 \\
0.1818\end{array}$ & $\begin{array}{l}-0.0430 \\
-0.0679 \\
-0.0242 \\
-0.0702\end{array}$ & $\begin{array}{l}0.008 \\
0.001 \\
0.008 \\
0.008\end{array}$ \\
\hline$\Delta n / \mathrm{cm}^{3} \mathrm{~mol}^{-1}$ & $\begin{array}{l}298.15 \\
303.15 \\
308.15 \\
313.15\end{array}$ & $\begin{array}{l}-25.8838 \\
-25.9433 \\
-25.5684 \\
-25.7260\end{array}$ & $\begin{array}{l}13.3282 \\
12.5385 \\
12.7389 \\
12.2372\end{array}$ & $\begin{array}{l}-6.9669 \\
-4.3289 \\
-6.1964 \\
-5.3558\end{array}$ & $\begin{array}{c}-0.8072 \\
4.7389 \\
3.3150 \\
4.3862\end{array}$ & $\begin{array}{l}0.053 \\
0.023 \\
0.016 \\
0.035\end{array}$ \\
\hline
\end{tabular}

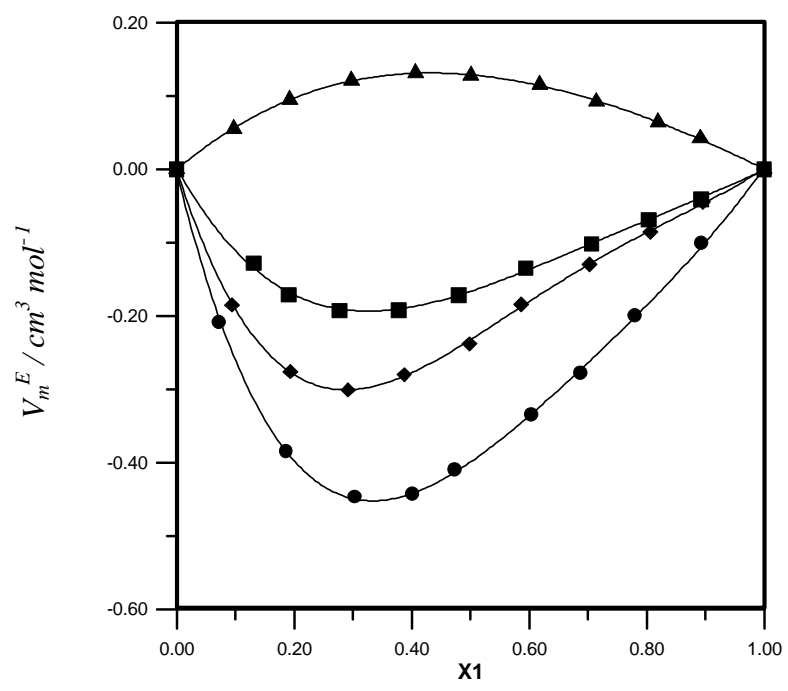

Fig.(1) Variation of excess molar volume $\left(V_{m}^{E}\right)$ against mole fraction $\left(x_{1}\right)$ of acetonitrile for the binary mixtures at $T=298.15 \mathrm{~K}$, acetonitrile + benzene ; $\checkmark$, acetonitrile + toluene ; $\bullet$, acetonitrile + m-xylene; $\mathbf{\square}$, acetonitrile +mesitylene; $\Delta$. 


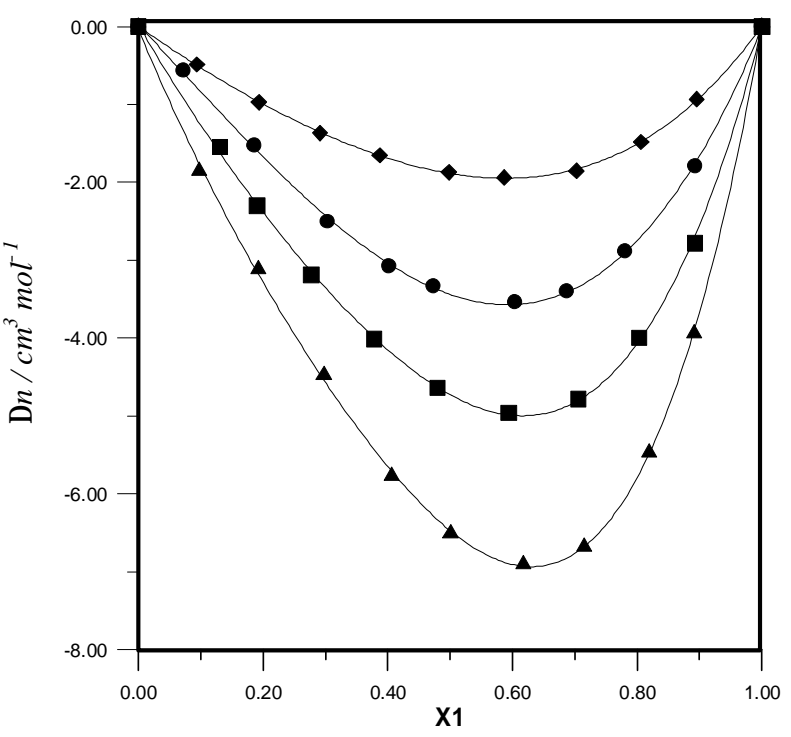

Fig.(2) Deviations in refractive index ( $\Delta n)$ versus mole fraction $\left(x_{1}\right)$ of acetonitrile for the binary mixtures at $T=298.15 \mathrm{~K}$. acetonitrile + benzene ; $\diamond$, acetonitrile + toluene ; $\bullet$, acetonitrile + m-xylene; $\square$, acetonitrile + mesitylene; $\Lambda$.

The results of excess molar volume vs mole fraction, $x_{1}$, of acetonitrile at $298.15 \mathrm{~K}$ are presented in (Fig.(1)) large negative $V_{m}^{E}$ is observed for the acetonitrile + toluene mixture, showing the presence of specific interactions. With increasing methyl group substitution on benzene such as in $m$-xylene and mesitylene the $V_{m}^{E}$ values become less negative, exhibiting the expansion in volume due to the presence of bulky methyl groups. Only in the case of acetonitrile + mesitylene is a large positive $V_{m}^{E}$ observed, which is attributed to the presence of weak dispersion type interactions.

The extent of deviation in $V_{m}^{E}$ from linear dependence on mole fraction (Fig.(1)) follows the sequence toluene $<$ benzene $<m$-xylene $<$ mesitylene. This suggests that there is an expansion in volume of the mixtures as we move from benzene to mesitylene. The difference in size and shape of the component molecules and the loss of dipolar association leads to expansion in volume. The physical interaction between unlike molecules such as donor-acceptor and dipole-dipole interactions results in contraction in volume.

It has been reported that the magnitude of $V_{m}^{E}$ is the result of different effects, which may be divided into physical, chemical, and structural contributions. Physical interactions involving mainly nonspecific interactions, contribute positively to $V_{m}^{E}$, and the breaking of the liquid order on mixing, also giving a positive contribution to $V_{m}^{E}$ while the chemical or specific interactions, such as complex formation and hydrogen bond formation between constituent molecules, contribute negatively to $V_{m}^{E}$; the structural effects rising from interstitial accommodation due to the difference in the molar volume and free volume between components also contribute negatively to $V_{m}^{E}$ [21].

The introduction of methyl groups in the ring will increase the electric donor-acceptor interaction. Hence toluene has a more negative excess volume than benzene. But the bulky methyl handles the proper orientation of acetonitrile to interact with $\mathrm{m}$-xylene and result in a little negative excess volume than toluene. Therefore, the $V_{m}^{E}$ values for $\mathrm{m}$-xylene should more than that for the toluene mixture. For m-xylene the specific interaction energy between the two unlike molecules is affected by the positive relation of two methyl groups in the ring. In general, the $V_{m}^{E}$ values increase as the number of - $\mathrm{CH} 3$ groups in the ring increase from benzene to mesitylene is 
due to the fact that the methyl group (-CH3) is an electron releasing group that would enhance the electron density of the benzene ring of the aromatic molecules; however, the electronaccepting tendency of the aromatic ring would decrease as we move from benzene to mesitylene, resulting in decreased donoracceptor interaction between unlike molecules with an increase in the number of methyl groups $(-\mathrm{CH} 3)$ in the aromatic hydrocarbon molecule, which would cause an expansion in the volume of the mixture. Another factor that would cause an increase in $V_{m}^{E}$ values is the steric hindrance due to - $\mathrm{CH} 3$ groups of the rings. As the number of methyl groups in the ring increase benzene to mesitylene, the closer approach of the acetonitrile molecule to the aromatic ring becomes increasingly difficult, resulting in decreased interaction between acetonitril and aromatic hydrocarbon molecules.

The values of $\Delta n$ vs $x_{1}$ at $298.15 \mathrm{~K}$ presented in (Fig.(2)) are negative for all mixtures, and their magnitudes decrease with increasing substitution of methyl groups on benzene. It may be noted that such values are due to the electronic perturbation of the individual molecules during mixing and, therefore, depend very much on the nature of the mixing molecules. The extent of negative deviation in $\Delta n$ from linear dependence on composition follows the sequence benzene $<$ toluene $<m$-xylene $<$ mesitylene. In general, the negative deviations in $\Delta n$ values indicate weak interactions between the components of the mixture [18]. The observed trends (Fig.2) of $\Delta n$ values indicate the presence of weak interactions in these mixtures, which follow the order benzene > toluene $>m$-xylene > mesitylene. The $\Delta n$ values decrease with the increase in temperature for each binary mixture, indicating that the interactions between unlike molecules weaken with a rise in temperature.

\section{Conclusions}

New experimental values of densities $(\rho)$ and refractive indices $\left(n_{\mathrm{D}}\right)$ of binary mixtures of acetonitrile with benzene, toluene, $m$-xylene and mesitylene at temperatures (298.15, $303.15,308.15$, and 313.15) $\mathrm{K}$ and different compositions are measured. The excess molar volumes $\left(V_{m}^{E}\right)$ and deviations in refractive indices $(\Delta n)$ were correlated using the Redlich-Kister polynomial equation. The excess molar volumes $V_{m}^{E}$ were negative for the acetonitrile, + benzene, + toluene, $+m$ xylene binary mixtures and positive for acetonitrile + mesitylene mixture at all temperatures and over the entire range of compositions. The deviations in refractive indices are negative and less negative with increasing temperature. The results are discussed in terms of the intermolecular interactions.

\section{References}

[1] Maravkova, L.; Linek, J. "Excess molar volumes of (benzene +isopropylbenzene, or 1, 3, 5-trimethylbenzene, or 1,2,4trimethylbenzene)at temperatures between 298.15 to $328.15 \mathrm{~K}^{\prime \prime}$ J J. Chem. Thermodyn. 35, 1139-1149, 2003.

[2] Giner, B.; Lafuente, C.; Villares, A.; Haro, M.; Lopez, M. C., "Volumetric and refractive properties of binary mixtures containing 1,4-dioxane and chloroalkanes"; J. Chem. Thermodyn. 39, 148-157, 2007.

[3] Nain, A. K.,"Refractive indices and deviations in refractive indices for binary mixtures of formamide +1 -butanol, +2 butanol, + 1,3-butanediol, and $+1,4-$ butanediol at temperatures from (293.15 to 318.15) K. "; J. Chem. Eng. Data. 53, 1208-1210, 2008.

[4] Mehta, S.K., Sharma, A.K., Bhasin, K.K., Prakash, R., " Physicochemical properties in mixtures of hexamethyl phosphortriamide with 2,2,2trichloroethanol or 2,2,2-trifluoroethanol or 1,1,1,3,3,3-hexafluoropropan-2-ol. " ; Fluid Phase Equilib. 201, 203-216, 2002.

[5] Zarei, H.A., Iloukhani, H., "Excess molar enthalpies of formamide + some alkan-1ols (C1-C6) and their correlations at 298.15 K. "; Thermochim. Acta. 405, 123-128, 2003.

[6] Absood.H.A,Tutunji.S.M, Hsu.Y.K., and Cleverge.L.H, "The Density and Enthalpy of Mixing of Solutions of Acetonitrile and of Dimethyl Sulfoxide with Several 
Aromatic Hydrocarbons"; J. Chem. Eng. Data, 21, 304-311,1976.

[7] Riddick, A. J.; Bunger, B.W. ; Sacano, K. T., "Organic Solvents. Physical Properties and Methods of Purification"; 4th edn. (Wiley, New York, 1986).

[8] T^orres,R. B.; Francesconi, A. Z.; Volpe, P. L. O. "Volumetric Properties of Binary Mixtures of Acetonitrile and Chloroalkanes at $25^{\circ} \mathrm{C}$ and Atmospheric Pressure"; J. Solution Chem. 32,417-434, 2003.

[9] Aminabhavi, T. M.; Bindu, G. "Density, Viscosity, Refractive Index, and Speed of Sound in Aqueous Mixtures of N,NDimethyl Sulfoxide,N,N-Dimethylacetamide, Acetonitrile, EthyleneGlycol, 1.4-Dioxane, Tetrahydrofuran, 2Methoxyethanol, and 2-Ethoxyethanol at 298.15K. " J. Chem. Eng. Data, 40, 856861, 1995.

[10] Yu, L.; Li, Y., " Excess Molar Volumes of Sulfolane in Binary Mixtures with Six Aromatic Hydrocarbons at 298.15 K"; Fluid Phase Equilib. 147, 207-213, 1988.

[11] Emmerling, U.; Figurski, G., "Densities and Kinematic Viscosities for the Systems Benzene + Methyl Formate, Benzene + EthylFormate, Benzene + Propyl Formate, and Benzene + Butyl Formate"; J. Chem. Eng. Data, 43, 289292, 1998.

[12] Aralaguppi, M. I.; Aminabhavi, T. M.; Harogoppad, S. B.; Balundgi,R. H. "Thermodynamic interaction in binary mixtures of dimethylsulfoxide with benzene, toluene, 1,3-dimethylbenzene, 1,3,5-trimethylbenzene, and methoxybenzene from 298.15 to 308.15 K."; J. Chem.Eng. Data, 37, 298-303, 1992.

[13] Exarchos, N. C.; Tasioula-Margar, M.; Demetropoulos, I.N., "Viscosities and densities of dilute solutions of glycerol trioleate + octane, $+\mathrm{p}$-xylene, + toluene, and + chloroform. "; J. Chem. Eng. Data,40, 567-571,1995.

[14] M.Singh; P. C. Gupta; R. N. Kesharwani, "Density and Viscosity for Mixtures of Propanoic Acid with
Aromatic Hydrocarbons at 298.15 K"; J. Chem. Eng. Data 40, 358-360,1995.

[15] Ali, A., Nain, A.K., Chand, D., Ahmad, R. "Viscosities and Refractive Indices of Binary Mixtures of Dimethylsulphoxide with Some Aromatic Hydrocarbons at Different Temperatures: An Experimental and Theoretical Study"; J. Chin. Chem. Soc., 53, 531-539, 2006.

[16] Moumouzias,G.; Ritzoulis, G., "Densities, Relative Permittivities, and Refractive Indices of the Binary Systems Propylene Carbonate $+\mathrm{O}$ Xylene and Propylene Carbonate $+\mathrm{m}$ Xylene at $(15,20,25,30$, and 35$){ }^{\circ} \mathrm{C} "$; J. Chem. Eng. Data, 45, 202-209,2000.

[17] Aralaguppi,M. I. ; Aminabhavi T. M., " Density, Refractive Index, Viscosity, and Speed of Sound in Binary Mixtures of Cyclohexanone with Benzene, Methylbenzene, 1,4-Dimethylbenzene, 1,3,5-Trimethylbenzene, and Methoxybenzene in the Temperature Interval (298.15 to 308.15) K"; J. Chem. Eng. Data, 44, 446-450, 1999.

[18] Brocos, P.; Pineiro, A.; Bravo, R.; Amigo, A. "Refractive indices, molar volumes and molar refractions of binary liquid mixtures:concepts and correlations. "; Phys. Chem. Chem. Phys., 5, 550-557, 2003.

[19] Redlich, O.; Kister, A. T. "Algebric representation of thermodynamic properties and classification of solutions"; Ind. Eng. Chem., 40,345348, 1948.

[20] Pal, A.; Dass, G. "Excess molar volumes and viscosities for binary liquid mixtures of methyl tert-butyl ether and of tertamyl methyl ether with methanol, 1propanol, and 1-pentanol at $298.15 \mathrm{~K}$, chlorobenzene, benzonitrile, and nitrobenzene". J. Chem. Eng. Data, 44, 1325- 1329, 1999.

[21] Matos, J. S.; Trenzado, J. L. "Volumetric Properties and Viscosities of the Methyl Butanoate + n-Heptane + n-Octane ternary system and Its Binary Constitutions in the Temperature Range from $283.15 \mathrm{~K}$ to $313.15 \mathrm{~K}$ "; Fluid Phase Equilib. 186, 207-234, 2001. 
تم في هذا البحث قياس الكثافة ومعامل الانكسار للمخاليط الثنائية المحتوية على الاسيتونايتريل مع البنزين,

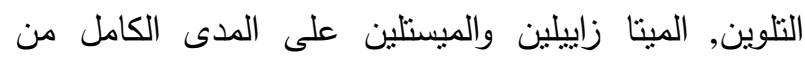
الكسور المولارية عند الدرجات الحرارية(298,15, 303,15, 308,15 و 303,15) كلفن والضغط الجوي.من قيم النتائج العملية للكثافة ومعامل الانكسار على التوالي تم حساب الحجوم المولارلية الفائضة( المولارية عن المثالية (هn). وتم تعييير القيم العملية لجميع

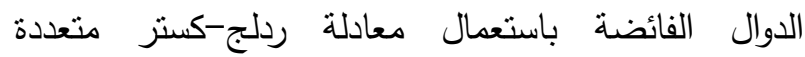
الحدود.اوضحت النتائج وجود تداخل ضعيف بين جزيئات الاسيتوناتريل والهيدروكاربونات الحلقية وقد اخذت قيم الحجوم

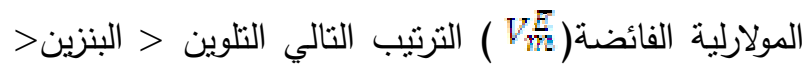
الميتا زاييلين > الميستلين. وقد فسرت قيم نتائج الدوال الفائضة على أساس التداخلات الجزيئية للنظام. 